\title{
Warped polar ring in the Arp 212 galaxy
}

\author{
A.V. Moiseev \\ Special Astrophysical Observatory Russian Academy of Sciences, Nizhnij Arkhyz, 369167, Russia \\ April 9, 2008/Revised: May 5, 2008

\begin{abstract}
The Fabry-Perot scanning interferometer mounted on the 6-m telescope of the Special Astrophysical Observatory of the Russian Academy of Sciences is used to study the distribution and kinematics of ionized gas in the peculiar galaxy Arp 212 (NGC 7625, III Zw 102). Two kinematically distinct subsystems - the inner disk and outer emission filaments - are found within the optical radius of the galaxy. The first subsystem, at galactocentric distances $r<3.5 \mathrm{kpc}$, rotates in the plane of the stellar disk. The inner part of the ionized-gas disk $(r<1.5-2 \mathrm{kpc})$ exactly coincides with the previously known disk consisting of molecular gas. The second subsystem of ionized gas is located at galactocentric distances $2-6 \mathrm{kpc}$. This subsystem rotates in a plane tilted by a significant angle to the stellar disk. The angle of orbital inclination in the outer disk increases with galactocentric distance and reaches $50^{\circ}$ at $r \approx 6 \mathrm{kpc}$. The ionized fraction of the gaseous disk does not show up beyond this galactocentric distance, but we believe that the HI disk continues to warp and approaches the plane that is polar with respect to the inner disk of the galaxy. Hence Arp 212 can be classified as a galaxy with a polar ring (or a polar disk). The observed kinematics of the ionized and neutral gas can be explained assuming that the distribution of gravitational potential in the galaxy is not spherically symmetric. Most probably, the polar ring have formed via accretion of gas from the dwarf satellite galaxy UGC 12549.
\end{abstract}

\section{Introduction}

Currently there is almost a consensus of opinion that gravitational interactions and mergers are among the most important factors in the evolution of galaxies, because such events change the observed structure, kinematics, starformation history, etc. Polar-ring galaxies (PRG) are one of the consequences of those interactions. Such objects exhibit rings or disks consisting of gas, dust, and stars, which rotate in the plane that is approximately perpendicular (polar) to the disk of the main ("host") galaxy. PRG are believed in most cases to owe their formation to galaxy mergers and accretion of the matter of the companion galaxy or gaseous filaments from the intergalactic medium onto the host galaxy (Bournaud \& Combes 2003. Combes (2006), and references therein).

PRG exhibit circular rotation in two mutually perpendicular planes thereby making it possible to study threedimensional mass distribution in the galaxy and determine the shape of its dark halo: oblateness, prolateness, and deviation from axial symmetry (Combes, 2006). To do this, it is necessary to obtain sufficiently detailed data about the inner kinematics of PRG. Whitmore et al. (1990) list 157 candidate polar-ring galaxies selected mostly by their peculiar appearance. However, the number of "real" PRG, i.e., which exhibit rotation in orthogonal planes, is much

Send offprint requests to: A.V. Moiseev, e-mail:

smaller. Even in the simplest case, where both the central galaxy and the ring are seen edge on, at least two longslit spectroscopic sections are needed to determine the rotation pattern of both subsystems. A number of problems arise in the cases where the spatial orientations of the galaxy and ring are such that one of the rotation planes is seen at a moderate angle to the line-of-sight. First, in many cases it is impossible to establish the PRG nature of an object solely from its direct images. Second, a substantially greater number of spectroscopic sections with different slit orientations are needed for a detailed kinematical study. In these cases it is better to determine the twodimensional velocity field using optical panoramic (3D) spectroscopy or radio interferometry in lines of molecular and atomic gas. The NGC 2655 galaxy is a good illustration. It was suspected to have a polar ring due to the powerful dust lane crossing the disk of the galaxy. A comparison of the velocity fields of gas and stars in the circumnuclear region confirmed such an interpretation (Sil'chenko \& Afanasiev, 2004), and recent 21-cm line observations allowed the structure and kinematics of the polar ring to be analyzed (Sparke et al., 2008). Note that for such complex objects even an extensive set of both photometric and kinematical data may be insufficient for a definitive conclusion about the structure of subsystems in the galaxy considered, see, e.g., the case of UGC 5600 (Shalyapina et al., 2007). 
In this paper we analyze new observational data for the peculiar galaxy Arp 212. Although this object was suspected to be a possible candidate PRG, no polar component has been found yet. In Section 2 we summarize the data related to this galaxy. In Section 3 we describe observations and reduction of the data obtained with the $6-\mathrm{m}$ telescope of the Special Astrophysical Observatory of the Russian Academy of Sciences. In Section 4 we show that the velocity field of ionized gas contains two subsystems with different rotation velocities. In the next Section 5 we construct a two-dimensional model of the rotation of gas in the outer regions to explain the kinematics of the polar ring. In Section 6 we discuss the overall structure of the galaxy and finally in Section 7 formulate the main results and conclusions of the paper.

\section{Arp 212: History of investigations}

Arp 212 (NGC 7625, III Zw 102) is a peculiar galaxy. According to the NED database, it belongs to SA(rs)a pec type and its optical diameter is $D_{25}=1$ !'6. Most of the authors classify this galaxy as an object of early morphological type - a lenticular or even an elliptical galaxy (see the discussion in Brosch \& Loinger, 1991). At the same time, the galaxy is rich in (both atomic and molecular) gas and contains numerous HII regions, which are indicative of violent star formation. According to Yasuda et al. (1992) and Li et al. (1993), the current star-formation rate lies in the interval from 2.7 to $8 M_{\odot} /$ year.

The high intensity of emission lines of ionized gas was one of the main reasons why Thuan \& Martin (1981) classified Arp 212 as a blue compact dwarf galaxy (BCDG), although according to Yasuda et al. (1992), its size $\left(D_{25}=11 \mathrm{kpc}\right)$, mass, and luminosity $\left((3-8) \times 10^{10} M_{\odot}\right.$, $\left.L_{B}=1.3 \times 10^{9} L_{\odot}\right)$, exceed substantially the corresponding parameters for dwarf galaxies. The tradition nevertheless persists and the object continues to appear in various catalogs and lists of BCD galaxies (Cairós et al., 2001a, Cairós et al.; 2001b, García-Lorenzo et al., 2008.

Some researchers also doubt the classification of Arp 212 as an early-type galaxy. Cairós et al. (2001a) show that even at small galactocentric distances $(r<1 \mathrm{kpc})$ the surface-brightness profile fits well the exponential law, which is typical of a flat disk. Therefore the above authors classify the galaxy as a late-type object (Im).

The most well-known feature of Arp 212, which shows up in all its published optical images, is the chain of dust lanes, which form an open ring with a radius of about $15-$ $20^{\prime \prime}$ extending along $P A \approx 45^{\circ}$. The Southwestern part of the ring stands out by its especially strong overlapping dust lanes. Because of this feature the galaxy not only appears in Arp's list of peculiar galaxies (Arp, 1966), but was also marked by Whitmore et al. (1990) as an object "related to polar-ring galaxies".

Another interesting structural feature was found on images taken in the $\mathrm{H} \alpha$ emission line (Cairós et al., 2001b). Most of the HII emission concentrates in the central part of the galaxy. At the same time, some of the HII regions are located in curvilinear segments extending out to $4 \mathrm{kpc}$ $\left(35-45^{\prime \prime}\right)$ from the center. Cairós et al. (2001b) believe that these features resemble the tidal tails in the wellknown Antennae galaxy (VV 245), i.e., they are due to recent interaction.

The kinematics of the interstellar medium in Arp 212 was studied repeatedly by many authors. Long-slit spectroscopy along the major axis of the galaxy revealed a significant gradient of line-of-sight velocity of ionized gas (Demoulin, 1969), the velocity of rotation amounts up to $155 \mathrm{~km} / \mathrm{s}$. Measurements of line-of-sight velocity of molecular gas (Yasuda et al., 1992) agree fairly well with the model of circular rotation of the gaseous disk. At the same time, appreciable deviations from circular rotation were found in several regions in the vicinity of dust lanespossibly due to impacts of gaseous clouds onto the disk of the galaxy. The rather low spatial resolution of these radio observations (beam $=15^{\prime \prime}$ ) made it impossible to unambiguously interpret the observed pattern of gas motions. The CO-line velocity field constructed by Li et al. (1993) with a twice higher resolution showed that the inner part of the molecular disk rotates circularly in the plane of the stellar disk. The above authors failed to measure the velocities of molecular gas in the region of dust lanes, but they report the HI velocity field and found the diameter of the HI disk to be four times greater than the optical diameter $D_{25}$. Note that outside the stellar disk gas rotates in the same plane, but in the opposite direction. The HI kinematics inside the optical disk is rather intricate with line-ofsight velocity contours turning almost by $90^{\circ}$. The above authors assumed that here we are dealing either with a strong warp of the gaseous disk, or a tilted gaseous ring. Unfortunately, the low spatial resolution (beam $\left.=51^{\prime \prime}\right)$ of their observations made it impossible to study the pattern of HI motions inside the optical disk.

There is no doubt that the galaxy bears signs of recent interaction, however, so far there are no clear answers to the following questions: what is the object the galaxy interacts with; how do the outer emission regions move and whether they belong to the galaxy at all, etc. The answers to these questions can be found by studying the velocity field of ionized gas in the "transition region" between the inner molecular disk and the outer HI structure. In 2003 Arp212 was observed with the scanning FabryPerot interferometer (FPI) attached to the 6-m telescope of the Special Astrophysical Observatory of the Russian Academy of Sciences (SAO RAS) at the request of MuñozTuñon (IAC, Spain) and her coathors. The primary aim of the project was to analyze the effect of intense star formation on the interstellar medium and we published the first results in our earlier paper Martínez-Delgado (2007). However, even a preliminary analysis of the velocity field allowed us to identify a second, independently rotating subsystem of ionized gas in Arp 212, which is indicative of a warped (and, possibly, polar) disk. The aim of this paper, which is based on archive observational data of the SAO RAS, is to study the kinematics of ionized gas in Arp 212. 
In accordance with $\mathrm{Li}$ et al. (1993), we assume that the distance to the galaxy is equal to $23.5 \mathrm{Mpc}$, which corresponds to an image scale of $115 \mathrm{pc} / 1^{\prime \prime}$.

\section{Observations and data reduction}

\subsection{Panoramic spectroscopy}

The Arp 212 galaxy was observed on November 30, 2003 with the scanning Fabry-Perot interferometer mounted inside SCORPIO focal reducer (Afanasiev \& Moiseev, (2005) located in the primary focus of the 6 -m telescope of the SAO RAS. The desired spectral interval in the neighborhood of the $\mathrm{H} \alpha$ line was cut using a narrow-band filter with a width of $F W H M=21 \AA$. The width of the free spectral interval between the neighboring orders of interference was equal to $13 \AA$ (about $600 \mathrm{~km} / \mathrm{s}$ ). The resolution of the interferometer $(F W H M$ of the instrumental profile) was $0.8 \AA(35 \mathrm{~km} / \mathrm{s})$ for a $0.36 \AA /$ channel scale. The detector was a $2048 \times 2048 \mathrm{EEV} 42-40 \mathrm{CCD}$ operating in the $4 \times 4$ pixel instrumental averaging mode in order to reduce the readout time. The resulting image scale and field of view were $0{ }^{\prime \prime} 7 /$ pixel and $6 ! 1 \times 6 ! 1$, respectively.

We took a total of 36 successive interferograms of the object with different gaps between the plates of the FabryPerot interferometer. The total exposure was $6480 \mathrm{~s}$ and seeing varied from 1". 3 to 1 ." 8 . To remove ghost images that appear in the plates of the Fabry-Perot interferometer employed, we observed the object successively in two fields turned in the position angle. We removed the ghost images using the algorithms described by Moiseev \& Egorov (2008). We reduced observational data using an IDL software package (Moiseev, 2002, Moiseev \& Egorov 2008). After primary reduction, night-sky line subtraction, and wavelength calibration the observations were reduced to the form of a data cube where each pixel in the $512 \times 512$ field of view contains a 36 -channel spectrum.

The final angular resolution corresponds to the 1 .'9 seeing. To increase the signal-to-noise ratio in low surface brightness regions, we smoothed the data cube with a two-dimensional Gaussian with the $F W H M$ equivalent to this seeing. With the above procedure applied the spatial resolution became equal to $22^{\prime \prime} 7$. We analyzed the data with both variants of spatial resolution and the resulting conclusions are mutually consistent. Below we give only the results obtained by analyzing the smoothed cube, because this a procedure yields somewhat more accurate kinematical parameters compared to those inferred from unsmoothed data.

We fitted the $\mathrm{H} \alpha$ emission profiles to the Voigt function, which in most cases describes the observed contour fairly well (see a discussion in Moiseev \& Egorov, 2008). The profile fitting results were used to construct the twodimensional field of line-of-sight velocities of ionized gas, the map of velocity dispersion, and images of the galaxy in the $\mathrm{H} \alpha$ line and in the neighboring continuum. We estimate the accuracy of line-of-sight velocity measurements by measured signal-to-noise ratios using the rela- tions given in Fig. 5 of Moiseev \& Egorov (2008). We found this accuracy to be $1-6 \mathrm{~km} / \mathrm{s}$ in bright HII regions and amount to $10-20 \mathrm{~km} / \mathrm{s}$ in regions with minimal surface brightness, where the emission line shows up at the signal-to-noise level of $S / N \approx 5$.

\subsection{Deep Image}

We took a direct image of Arp 212 on February 5, 2008 with the 6-m telescope of the SAO RAS using the same instrument (SCORPIO focal reducer). The total exposure in the $\mathrm{R}_{c}$ filter of the Johnson-Cousins system was equal to $1320 \mathrm{~s}$, the seeing was $1^{\prime \prime} .9$, the image scale $0.35 /$ pixel and the field of view $! 1 \times 6 ! 1$. We calibrated the image in to magnitudes using the surface-brightness profile obtained by Cairós et al. (2001a) in the same filter. Although observations were made under nonphotometric atmospheric conditions at zenith distances $z=61-71^{\circ}$, we managed to obtain so far deepest available image of the galaxy. The limiting detection magnitude at the $1 \sigma$ level is equal to $25.2-25.5^{m} / \square^{\prime \prime}$. The visible radially averaged surfacebrightness profile extends one and a half times farther from the center of the galaxy compared to the profile obtained by Cairós et al. (2001a) and reaches a surface brightness level of $26^{m} / \square^{\prime \prime}$. The image was taken in order to search for eventual low surface brightness tidal features in the vicinity of the galaxy. However, it is evident from Fig. 1 that no external filaments show up in the field of view.

We decomposed the surface-brightness profile into standard components: exponential disk (with a radial scale $h=10^{\prime \prime} 3$, which is close to the result of Cairós et al. (2001a), and a central surface brightness of $\mu_{0}=17.4^{m}$ ) and Sérsic's bulge with parameters $n=1, r_{e}=5{ }^{\prime \prime} 2$, and $\mu_{e}=17.7^{m}$. The central surface brightness of the disk far exceeds the average central surface brightness for disk galaxies, as it is typical for interacting systems (Reshetnikov et al.,1993). The components luminosity ratio $B / D=0.20$ allows us to classify the galaxy as a late-type system $(\mathrm{Sc})$. Note that because the exponent in Sérsic's law is $n=1$ and the inclination of the galaxy is small, we cannot distinguish the bulge from an inner exponential disk and therefore the contribution of the spherical component may be even smaller and the morphological type of the galaxy may be even later.

\section{Analysis of the line-of-sight velocity field}

\subsection{Circular Rotation Model}

In Fig. 2 one can see the $\mathrm{H} \alpha$ image of the galaxy and the line-of-sight velocity field of ionized gas. We confidently measured the line-of-sight velocities in almost all outer emission regions found by Cairós et al. (2001b). It is evident from the figure that the form of line-of-sight velocity contours corresponds to the circular rotation in the region within $r \approx 20^{\prime \prime}$, i.e., where most of the $\mathrm{H} \alpha$ emission is concentrated. The pattern of the line-of-sight velocity field 


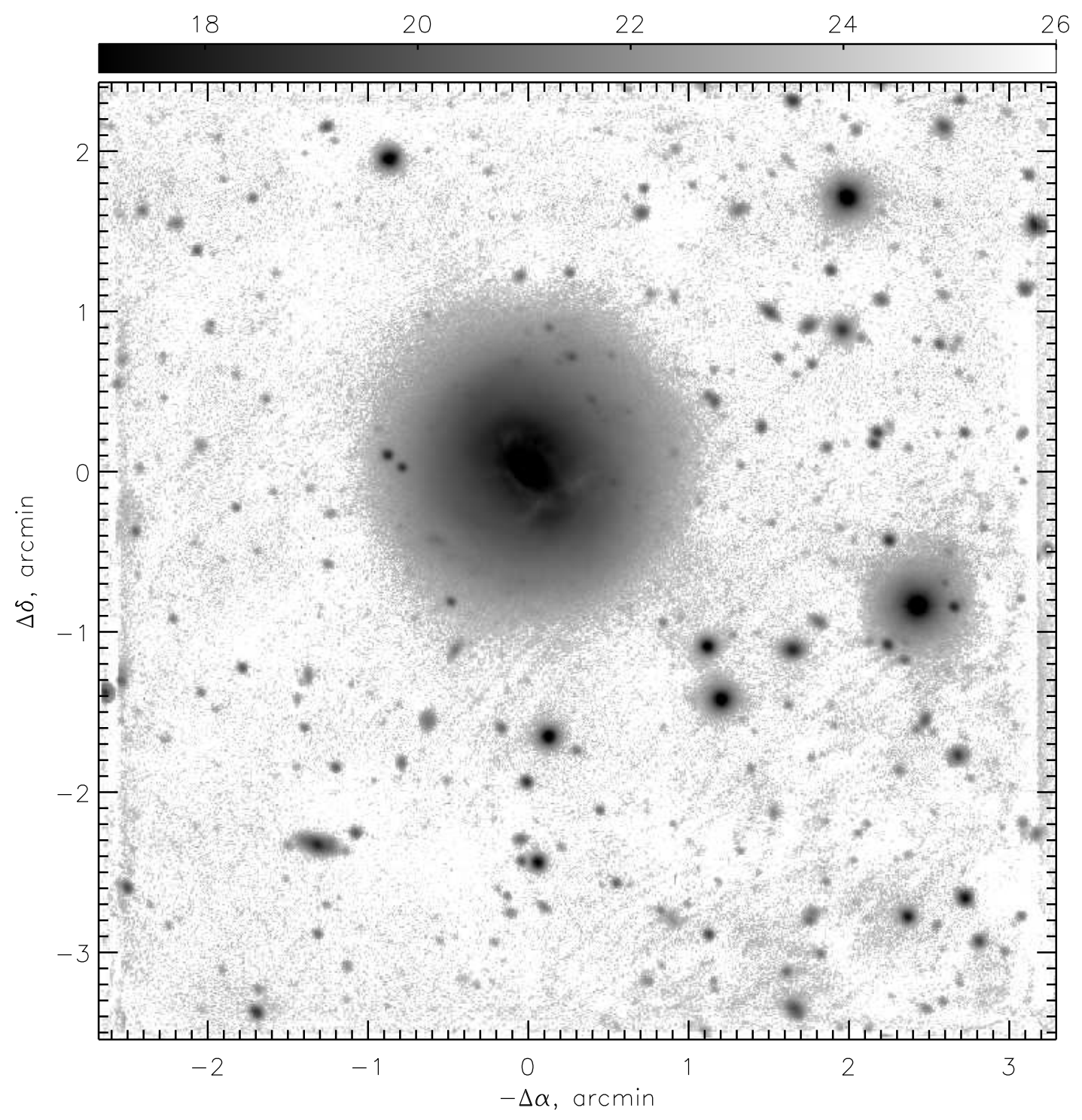

Fig. 1. Deep $\mathrm{R}_{c^{-}}$-band image of Arp 212 in magnitude scale.

agrees well with the results of integral-field spectroscopy for the inner region reported by García-Lorenzo et al. (2008). The location of the center of rotation as inferred assuming that the velocity field is symmetric agrees within the errors with the center of the $\mathrm{H} \alpha$ and continuum brightness contours. We analyzed the velocity field using the method of "tilted rings" Begeman (1989). We subdivided the velocity fields into $1^{\prime \prime}$-wide elliptical rings oriented according to the adopted position angle of the major axis $\left(P A_{0}\right)$ and inclination of the disk $\left(i_{0}\right)$. We fixed the position of the center of rotation. In the first approximation we estimated $P A_{0}$ and $i_{0}$ from photometric results. We then determined in each ring the optimum position angle of the kinematical axis $P A_{k i n}$, the mean velocity of rotation $V_{\text {rot }}$ and systemic velocity $V_{\text {sys }}$. For the justification of the method and references to the original works, see, e.g., Moiseev \& Mustsevoi (2000) and Moiseev et al. (2004).

Our preliminary analysis revealed two groups of domains in the velocity field: domains with velocities close to those implied by the circular-rotation model at the given galactocentric radius $r$, and domains with velocities that appreciably differ from the circular-rotation velocities by more than a certain threshold value $\left(v_{l i m}\right)$. We constructed several variants of the circular-rotation model choosing $v_{\text {lim }}$ so as to make data points belonging to each velocity group concentrate in spatially connected domains in the sky plane. We found that the best result is achieved with $v_{l i m} \approx 36 \mathrm{~km} / \mathrm{s}$. In this case two types of domains show up conspicuously in Fig. 33 the inner disk with close-to-circular rotation and isolated kinematically distinct domains. The latter include all HII regions 

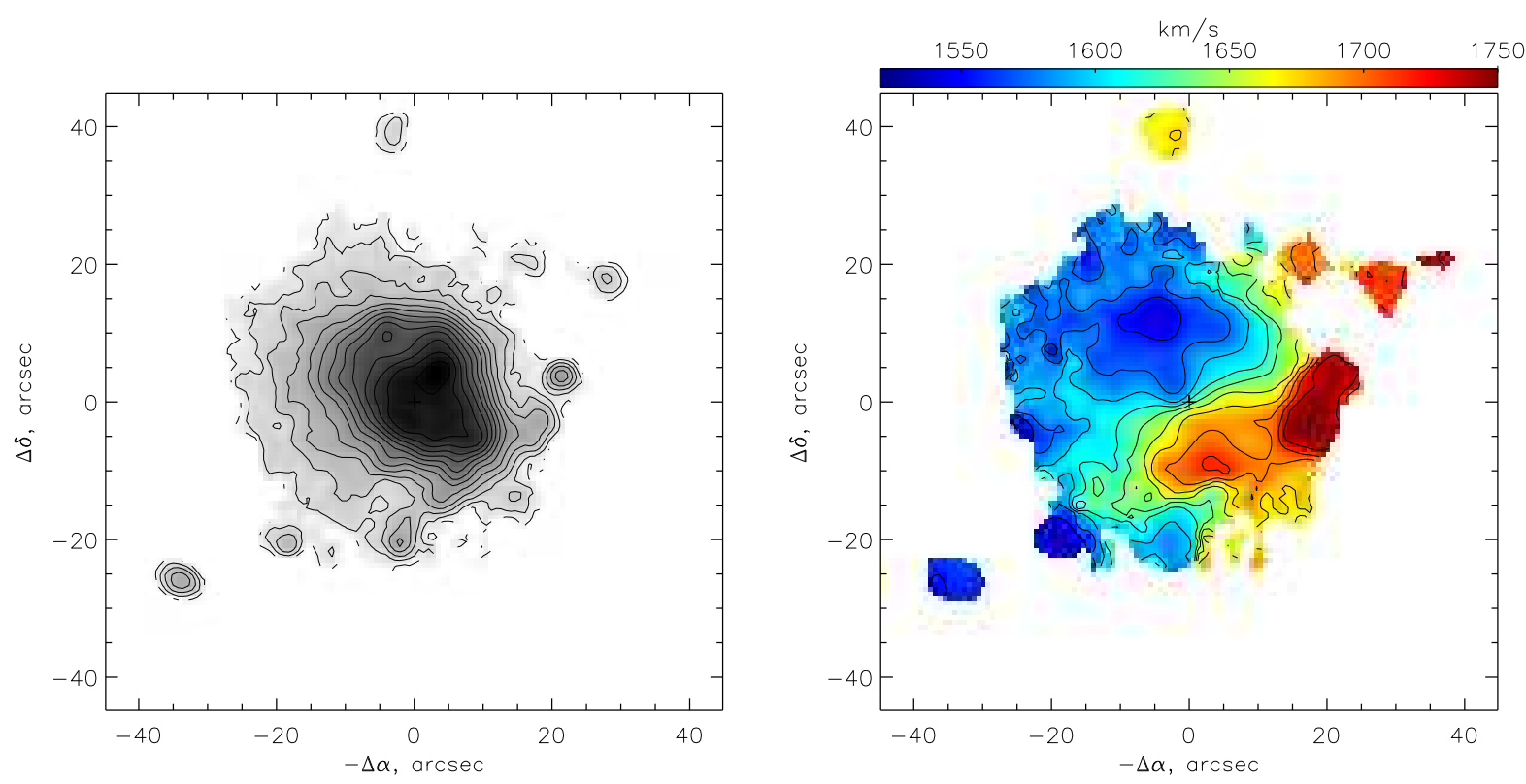

Fig. 2. Results of $\mathrm{H} \alpha$ observations of Arp 212: intensity distribution in logarithmic scale (left panel) and line-of-sight velocity field (right panel). The coordinate origin coincides with the center of continuum isophotes.

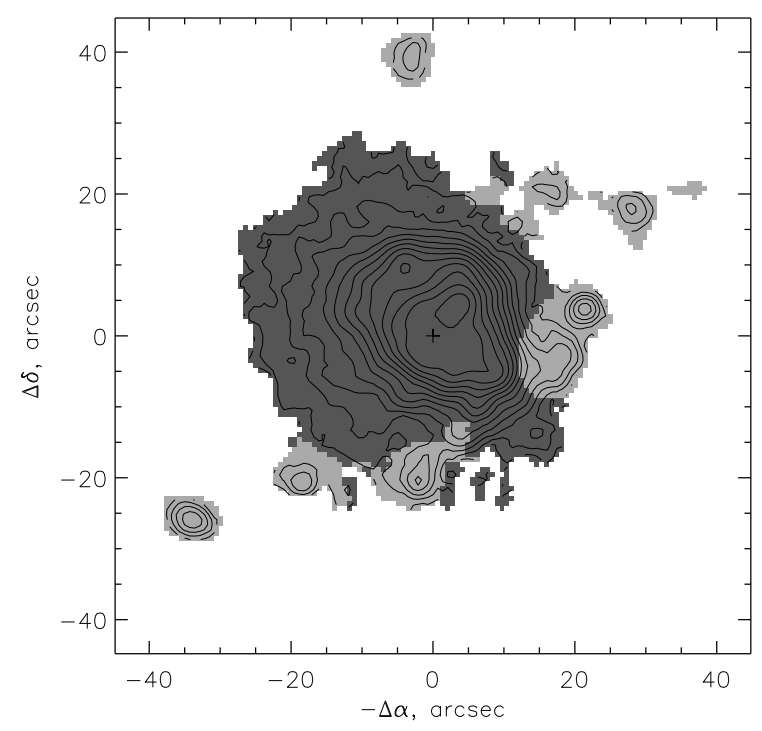

Fig. 3. The subdivision of galaxy into domain of the inner disk (dark shaded) and outer filaments (light shaded) with $\mathrm{H} \alpha$-line isophotes superimposed.

at galactocentric distances greater than $20^{\prime \prime}$ and several HII regions projected onto the Southwestern edge of the disk of the galaxy.

Table 1 lists the orientation parameters for the inner disk of the galaxy inferred from the velocity field assuming that $P A, i$, and $V_{\text {sys }}$ remain constant along the radius (the model of purely circular rotation in a fixed plane). These parameters agree within the $3 \sigma$ with those found by $\mathrm{Li}$ et al. (1993) for the velocity field of the molecular gas disk at $r \leq 15^{\prime \prime}$.

\subsection{Quasi-circular rotation model}

\subsubsection{Inner disk}

We refined the above model using the quasi-circular approximation, which allows noncircular motions, disk warp, etc. to be taken into account. We assumed that gas rotates in circular orbits whose $V_{\text {sys }}$ and $P A_{k i n}$ vary with $r$. Results of the analysis are presented in Fig. 4. Systemic velocity does not deviate from the mean value determined above by more than $\pm 10 \mathrm{~km} / \mathrm{s}$. The rotation curve of the inner part of the disk is close to the molecular-gas data, adjusted to our adopted value for inclination $i_{0}$. However, there are also certain systematic differences. First, our rotation curve shows a much sharper gradient of the rotation velocity of ionized gas, which must be due to the 2.5 times lower resolution of radio observations of $\mathrm{Li}$ et al. (1993). Second, the maximum rotation velocity of the molecular gas is somewhat higher than the rotation velocity of the ionized gas, although two velocities agree within the measurement errors. This difference may be due to asymmetric drift, i.e., to different velocity dispersions of the components studied. Indeed the velocity dispersion of ionized gas outside $r>10^{\prime \prime}$ exceeds $50 \mathrm{~km} / \mathrm{s}$, and such a behavior is indicative of the additional increase of chaotic motions caused by a powerful burst of star formation (MartínezDelgado, 2007).

A systematic increase of $P A_{k i n}$ can be seen in the interval $r=13-30^{\prime \prime}$ and the total amplitude of its variations exceeds $30^{\circ}$. This phenomenon can be most naturally explained by the warp of the outer disk regions. 
Unfortunately, because of the rather small $i_{0}$ the method employed is unstable with respect to parameter $i(r)$ - the tilt of the disk with respect to the sky plane inside each narrow ring. Therefore the adopted model cannot be used to determine the variation of the tilt of the warped part of the disk with respect to the line-of-sight.

\subsubsection{Outer regions}

On the left panel of Fig. 5 we present the map of residual velocities (observed minus model). Here we extrapolated the model up to outer filaments. Residual velocities in the inner disk mostly do not exceed $\pm 20 \mathrm{~km} / \mathrm{s}$, whereas in the outer HII regions they are several times higher and exhibit well-defined systematic behavior varying smoothly from $+100 \mathrm{~km} / \mathrm{s}$ in the Northwest to $-75 \mathrm{~km} / \mathrm{s}$ in the Southeast. The pattern of the velocities of outer filaments resembles the rotation of an inclined disk with orientation parameters differ appreciably from those of the inner region.

We determined the parameters of the rotation model for outer regions in terms of a purely circular-rotation model like we did it above for the inner disk. We list these parameters in the last column of Table 1. The systemic velocities of both kinematic components coincide, whereas their $P A_{0}$ and $i_{0}$ differ substantially. The center of rotation of outer regions is located $1^{\prime \prime}$ from the center of the inner disk, i.e., both centers coincide.

The diamond signs in Fig. 4 show the results of our analysis of the velocity field of filaments in terms of quasicircular approximation. We performed our analysis assuming that angle $i$ remains constant along the radius. We again failed to find the variations of $i(r)$ due to rather small number of data points inside each narrow ring. The position angle of the line of nodes for circular orbits of filaments varies with galatcocentric radius so that in the outer regions the turn of $P A$ with respect to the inner disk amounts to $90^{\circ}$ and exceeds appreciably the $P A_{0}$ value listed in Table 1, The variations of $P A_{k i n}$ about its mean value are usually interpreted either as signature of noncircular motions in the disk plane or as manifestations of the orientation (the warp) of the disk with respect to the lineof-sight. We believe the former hypothesis to be unlikely, because a turn of $P A_{k i n}$ by several tens of degrees would require substantial radial and azimuthal motions (exceeding $50 \mathrm{~km} / \mathrm{s}$ ), which must be comparable to the velocity of circular motion. Such a situation is sometimes observed in barred galaxies, but it is by no means typical of outer disk regions, since it require a substantial asymmetry in the distribution of gravitational potential (spiral density waves and a bar). We believe the second interpretation (the turn of the plane of circular orbits) to be most realistic, since the warp of the outer gaseous disk is a common phenomenon, especially in interacting galaxies. Below we consider the possible orientations of the warped disk with respect to the plane of the galaxy. flushleft

Table 1. Average kinematical parameters of gaseous subsystems.

\begin{tabular}{lrr}
\hline \hline Parameter & $\begin{array}{r}\text { Inner disk } \\
\left(\text { at } r=0-20^{\prime \prime}\right)\end{array}$ & $\begin{array}{r}\text { External parameters } \\
\left(\text { at } r=25-50^{\prime \prime}\right)\end{array}$ \\
\hline$P A_{0}\left(^{\circ}\right)$ & $211 \pm 6$ & $275 \pm 15$ \\
$i_{0}\left({ }^{\circ}\right)$ & $32 \pm 8$ & $60 \pm 10$ \\
$V_{\text {sys }}(\mathrm{km} / \mathrm{s})$ & $1627 \pm 5$ & $1626 \pm 6$ \\
\hline
\end{tabular}

flushleft

Table 2. Parameters of the two-dimensional model.

\begin{tabular}{lr}
\hline \hline Parameter & Value \\
\hline$\delta_{0}\left({ }^{\circ}\right)$ & $29 \pm 3$ \\
$\delta_{1}\left({ }^{\circ} /{ }^{\prime \prime}\right)$ & $0.40 \pm 0.06$ \\
$\gamma_{0}\left({ }^{\circ}\right)$ & $51 \pm 5$ \\
$\gamma_{1}\left({ }^{\circ} /{ }^{\prime \prime}\right)$ & $0.94 \pm 0.15$ \\
$V_{\max }(\mathrm{km} / \mathrm{s})$ & $89 \pm 7$ \\
$R_{h}\left({ }^{\prime \prime}\right)$ & $-46 \pm 25$ \\
$V_{\text {sys }}(\mathrm{km} / \mathrm{s})$ & $1627 \pm 1$
\end{tabular}

\subsection{Spatial Orientation of Orbits}

The significant differences between the orientation parameters of the inner and outer gaseous subsystems (Table 1) indicate that the rotation plane of outer filaments is tilted substantially to the plane of the inner disk. Angle $\delta$ between the two planes is given by the scalar product of their directing vectors via the following formula:

$\cos \delta= \pm \cos \left(P A_{2}-P A_{1}\right) \sin i_{1} \sin i_{2}+\cos i_{1} \cos i_{2}$.

Here subscripts " 1 " and " 2 " indicate the observed orientation parameters of both disks. The sign ambiguity in the first term is due to the fact that the position angle of the major axis, $P A$, and inclination $i$ as determined via kinematical analysis do not fully characterize the position of the plane with respect to the observer - we must know which side is the closest and which is the farthest from us. For the ambiguity to be resolved, the formula should be written as:

$\cos \delta=\cos \left(P A_{2}-P A_{1}\right) \sin i_{1} \sin i_{2}+\cos i_{1} \cos i_{2}$,

specifying how the inclination $i$ should be counted and taking into account the direction of rotation of the disks. If the vector of angular momentum is directed toward the observer, then $i<90^{\circ}$, if the angular momentum points to the opposite direction, then the inclination is defined as $180^{\circ}-i$.

The dust lanes mentioned in Section 2 and the dust ring are most conspicuous in the Southern and Southeastern parts of the galaxy disk (see the image shown in Fig. 1). The color-excess maps (Cairós, 2001b) also indicate that the Southeastern part of the galaxy stands out because of its redder color, which is due to the dust 

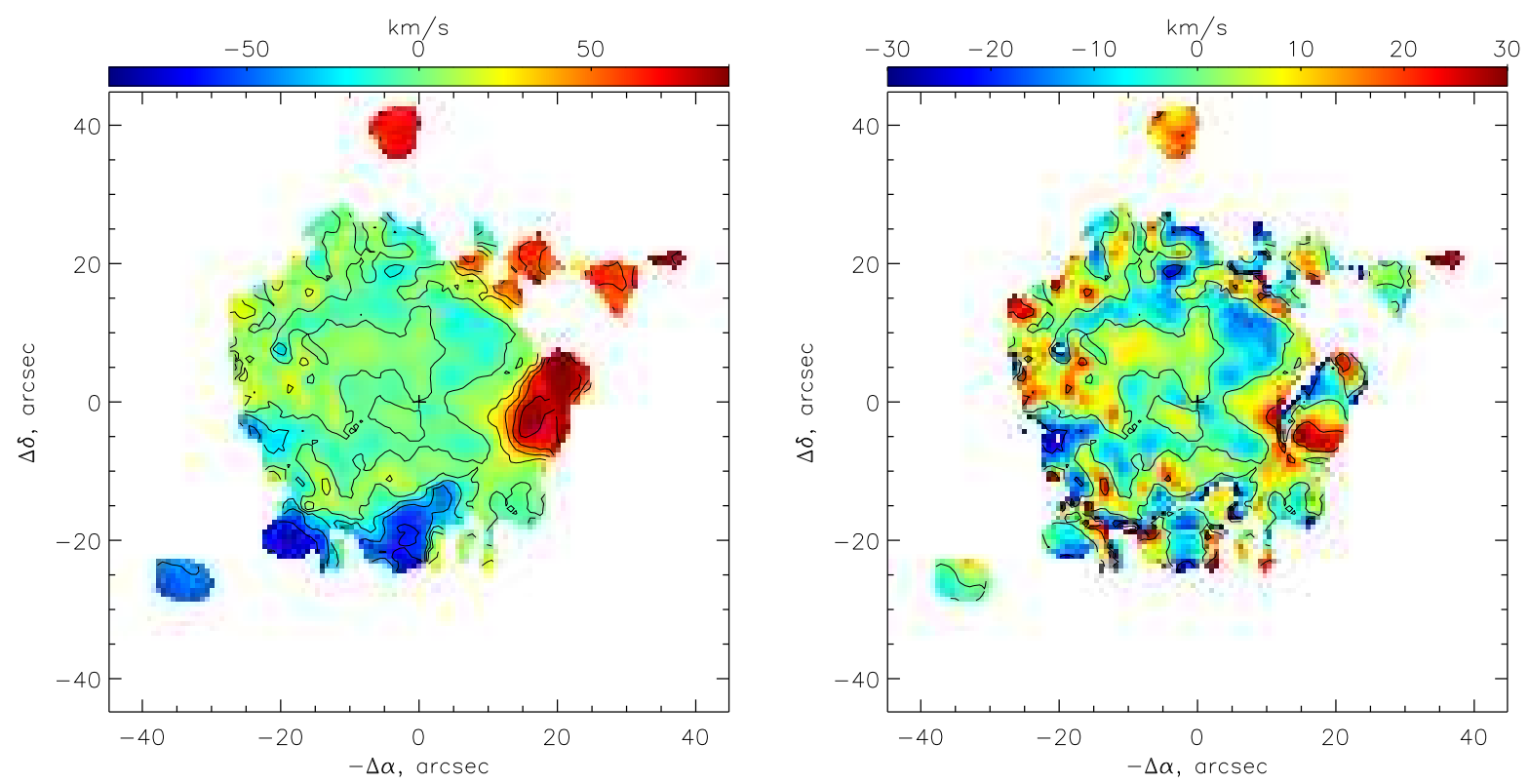

Fig. 5. Maps of the residual line-of-sight velocities (observed minus model): cases of a circular rotation model (left panel) and separate accounting for the rotation of inner and outer regions (right panel).

environment. This conclusion is confirmed by the Balmerdecrement maps reported by García-Lorenzo et al. (2008). The observed asymmetry of the distribution of extinction implies that the Southeastern part of the stellar disk of Arp 212 is the closest to us, and the pattern of the velocity fields implies that the vector of angular momentum is directed toward us.

It is evident from Fig. 3 that most of the external emission regions concentrate in the Southeast part of the galaxy, whereas there are no anomalous (compared to HII regions of the inner disk) HII regions in the position-angle interval $P A \approx 0-130^{\circ}$. It is safe to assume that this part of the outer disk is hidden from the observer by the stellar disk of the galaxy. In this case the Southeastern part of the orbits of emission filaments is the closest to us and the angular-momentum vector is directed toward the observer.

In view of the above discussion of the orientation of the disk with respect to the observer we use Eq. (1) to compute the angle between the two planes for $i_{1}=32^{\circ}$, $i_{2}=60^{\circ}, P A_{1}=211^{\circ}$ assuming that $P A_{2}$ varies with radius as shown in Fig. 4. Namely, $\delta=(45 \pm 11)^{\circ}$ for the inner part of the warped disk $\left(r=20^{\prime \prime}\right)$, whereas the angle increases with galactocentric distance and reaches $(68 \pm$ $10)^{\circ}$ at $r=50^{\prime \prime}$.

\section{2D model}

The above investigation of the kinematics of gas performed using the method of "tilted rings" constitutes the traditional approach toward the analysis of the velocity fields of galactic disks. Although we managed to obtain a number of quantitative estimates and conclusions concerning the behavior of outer gaseous filaments, this approach has nevertheless a certain disadvantage. Because of the insufficient sampling of the velocity field by data points at different position angles we had to assume that the tilt of orbits in the outer disk with respect to the line-of-sight does not vary with radius. At the same time, we showed that this disk is appreciably warped, so that the inclination of orbits with respect to the inner regions varies with radius. To better analyze the behavior of such a disk, we used the two-dimensional model of a warped disk described by Coccato et al. (2007). In this model the disk is subdivided into narrow rings and the orientation of $n$-th ring with respect to the main plane of the galaxy is specified by vertical angl $11 \delta_{n}$ and azimuthal angle $\gamma_{n}$. We assume that these angles vary linearly with galactocentric distance $r_{n}$ :

$\delta_{n}\left(r_{n}\right)=\delta_{0}+\delta_{1} r_{n}, \quad \gamma_{n}\left(r_{n}\right)=\gamma_{0}+\gamma_{1} r_{n}$

For the rotation curve of the disk we adopt the following widely used approximation (Courteau, 1997; Coccato, 2007):

$V_{\text {rot }}\left(r_{n}\right)=\frac{2}{\pi} V_{\text {max }} \arctan \frac{r_{n}}{R_{h}}$.

Formulae (2) and (3) approximate fairly well the results obtained in terms of the model described in the previous section.

For each ring we compute the distribution of lineof-sight velocities in accordance with rotation curve (3). All the necessary formulae can be found in (Coccato, 2007). The contribution of each ring to the total velocity field was assumed be the same for all rings. Unlike

\footnotetext{
1 For the sake of convenience, we maintain, where possible, the designations adopted by Coccato et al. (2007).
} 
Coccato et al. (2007), we did not simultaneously approximate the surface-brightness distribution of the warped disk because of its "clumpy" appearance.

This approach fundamentally differs from the methods described in Section 4 here we construct the model for the entire velocity field and fit it to observations. We fold the model velocity field with the two-dimensional Gaussian with a half-width corresponding to the spatial resolution of our data.

The $\chi^{2}$ minimization yields seven parameters, which fully characterize the warped disk: $\delta_{0}, \delta_{1}, \gamma_{0}, \gamma_{1}, V_{\max }$, $R_{h}$, and systemic velocity $V_{\text {sys }}$. We developed WARPWID software package for modeling and used MPFIT library written by B. Markwardt2 for nonlinear minimization. As the initial approximation for the parameter values we used the estimates obtained in terms of the model of "tilted rings" described in Section 4 . We list the resulting parameter values in Table 2. The quoted errors correspond to $3 \sigma$ deviations. Multiple runs with slightly different parameters showed that the solution obtained is stably reproducible and depends little on the initial conditions.

In Fig. 6] we represent the model velocity field and the field of residual line-of-sight velocities for the outer filaments. The deviations of observed velocities from the corresponding model velocities are somewhat smaller than in Fig. 5. The only exceptions are the inner boundaries of two HII regions South of the nucleus, where residual velocities exceed $25-30 \mathrm{~km} / \mathrm{s}$. These data points most likely correspond to the transition region where the velocities of the inner disk and outer HII regions are projected simultaneously onto the line-of-sight.

The solid lines in Fig. 4 4 reflect the radial dependences of the kinematical parameters computed in terms of our two-dimensional model: the rotation curve, position angle of orbits in the warped disk, the inclination of the orbits with respect to the sky plane, and systemic velocity (which is constant along the radius). It is evident from the figure that $V_{\text {sys }}$ and $P A$ agree with the results obtained in terms of the approximation of "tilted rings" 3 within the quoted errors. The rotation velocity in the two-dimensional model varies with radius, but it is about $30 \%$ higher than the velocity inferred in terms of the approximation of "tilted rings". Correspondingly, the inclination of circular orbits to the sky plane in case of the two-dimensional model differs substantially from that adopted in Section 4 -it appreciably decreases with radius. Note that the angle between the inner and warped disks is, according to Table 2 . equal to $\delta=(37 \pm 3)^{\circ}$ at $r=20^{\prime \prime}$ and reaches $(49 \pm 4)^{\circ}$ at $r=50^{\prime \prime}$. Thus in the two-dimensional model this angle

2 The most recent version of MPFIT is available at http://cow.physics.wisc.edu/ craigm/idl/idl.html

3 Note that model dependences shown by the lines in Fig. 4 are not approximations of the results of computations made in terms the "tilted rings" approximation. So, in each of the models considered in Sections 4 and 5 the same sky-plane point corresponds to different galactocentric distances $r$ in 3D space, since the orientations of orbits differs between these two models. also increases with distance, albeit somewhat slowly than in case of the "tilted rings" approximation.

Let us now point out the main difference between the kinematical models considered. The approximation of "tilted rings" requires more than 30 parameters to describe the velocity fields of outer emission filaments: the common inclination $i$ and three parameters $\left(P A_{k i n}, V_{r o t}\right.$, $\left.V_{\text {sys }}\right)$ for each narrow ring. The small number of points with measured velocities inside each ring and their nonuniform distribution in position angle make it is impossible to measure the variations of $i$ with radius and the resulting model is unstable. In the two-dimensional model the velocity field is described only by seven parameters. This model is much more stable, but its weak point is use of a priori formulae for the rotation curve (21) and the law of variation of orientation angles (3). These formulae were proposed based on the results of the analysis performed using the method of "tilted rings", i.e., our two-dimensional model is actually the next approximation to the description of the kinematics of the gaseous disk, which refines and supplements the results reported in Section 4 . That is why below when discussing the kinematics of the outer gaseous disk we refer to the results obtained in terms of the two-dimensional model, since we consider it to be more realistic.

\section{Discussion}

\subsection{Three-dimensional structure of the gaseous subsystem of Arp 212}

The facts that radial velocities of the outer disk agree well with the circular-model approximation (which takes into account the warp of the plane of rotation) and the systemic velocity coincides with the systemic velocity for the inner disk, indicates that we observe steady-state gas rotation. This would not be the case of the emission regions mentioned above belonged to a tidal tail as suggested by Cairós et al. (2001b). In Fig. 7 the sky-plane projections of orbits both for the inner disk $\left(r<25^{\prime \prime}\right)$ in the plane of the galaxy, and for outer regions $\left(r=20-50^{\prime \prime}\right)$ are presented. Both types of subsystems - the inner flat disk and the outer warped disk - coexist in the distance interval $r=20-30^{\prime \prime}(2.0-3.5 \mathrm{kpc})$ and hence their orbits must intersect. The collisions of gaseous clouds are possible in the region of intersections. It is immediately apparent from the right-hand panel of Fig. 7 that the powerful dust ring on the image of the galaxy is located inside these radii. The dust ring must correspond to the region where the gaseous disks collide. In this region increase of gas density behind shock fronts results in the formation of molecular and dust clouds like it happens in the Galactic spiral shock waves. An analysis of the ionization state of the inner regions of the galaxy also points to a possible contribution of shocks into the ionization of gas at $r \geq 15^{\prime \prime}$, where the dust ring starts García-Lorenzo (2008).

Perhaps it would be more correct to view the process not as direct collision, but as the "fall" of gas from the 

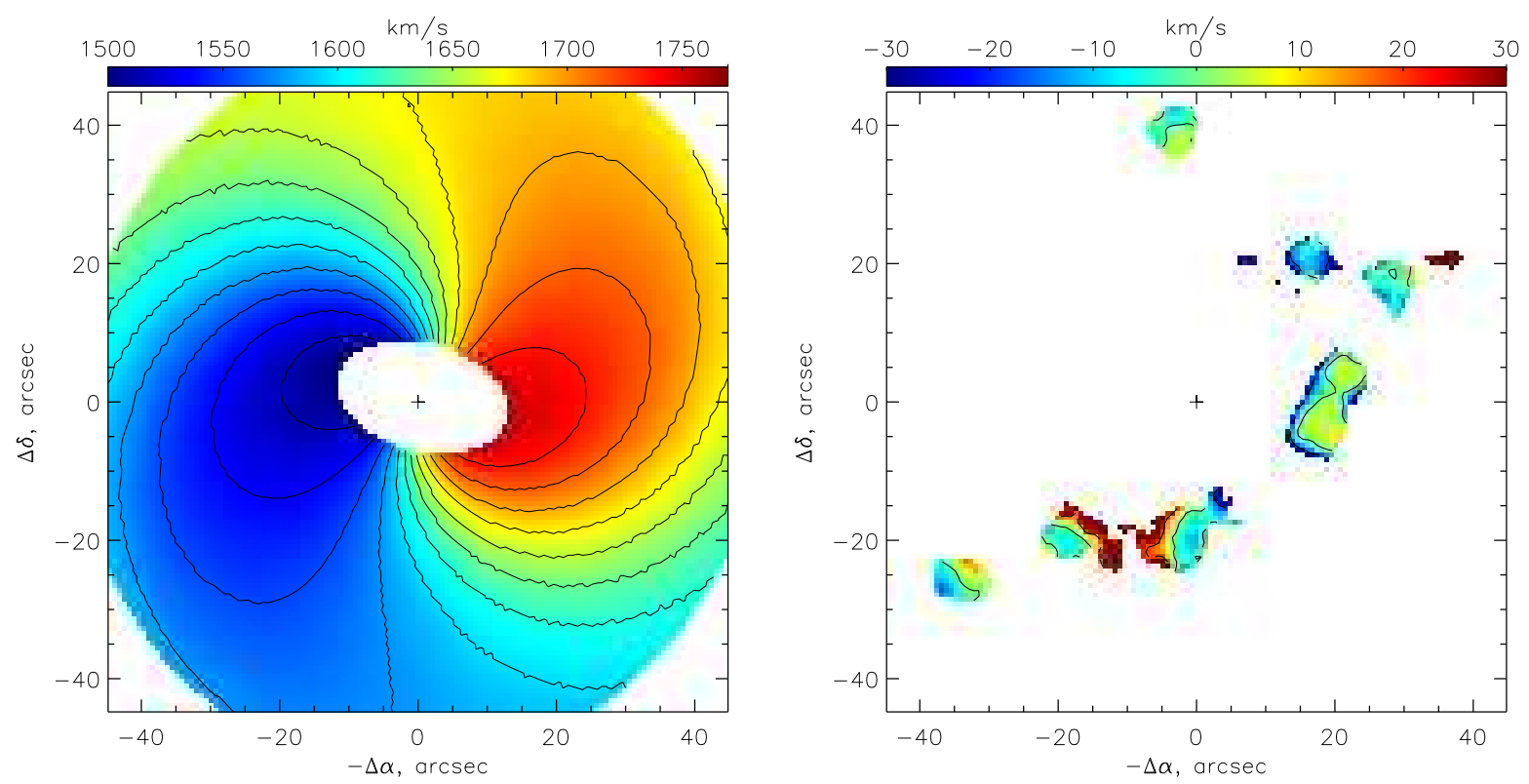

Fig. 6. Velocity field in the 2D warped-disk model (left panel) and the field of residual line-of-sight velocities in outer filaments (right panel).
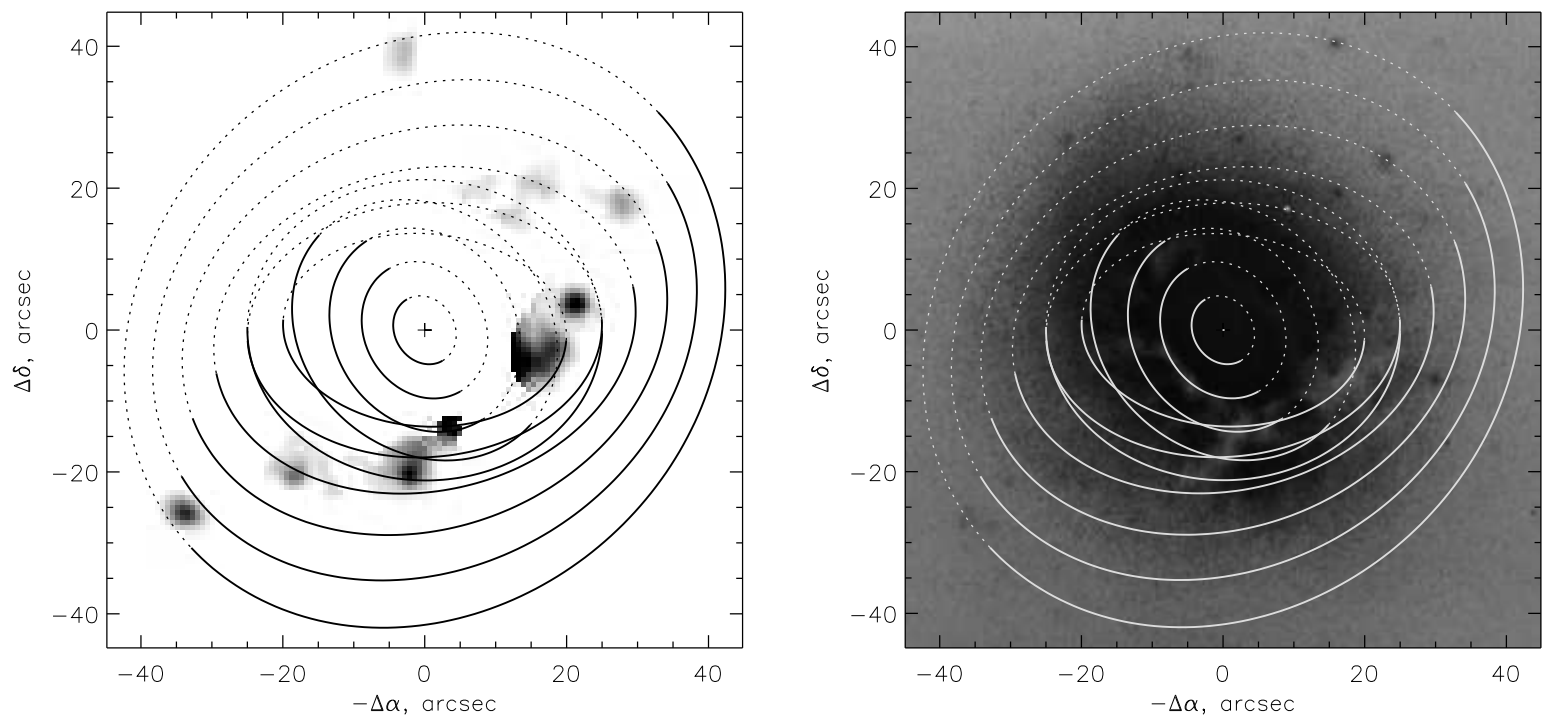

Fig. 7. $\mathrm{H} \alpha$ image of the regions located in the warped outer disk of the galaxy (left panel) and the red-plate photograph of the galaxy from Arp's (1966) atlas (right panel). The ellipses indicate the projections of circular orbits (with a radial step of $\left.5^{\prime \prime}\right)$. The dashed lines indicate the parts of the orbits located behind the sky plane. Both the orbits in the inner disk (in the plane of the galaxy) and those in the outer warped disk (the ring) are shown.

outer warped disk onto the main disk of the galaxy, because the angle between the two disks decreases toward the center, i.e., precessing orbits of gaseous clouds in the outer disk approach the stellar disk of the galaxy and clouds fall onto this stellar disk.

When referring to the gaseous subsystem to which the outer emission regions belong we usually employed the term "the warped disk". However, strictly speaking, we are dealing with a "ring", because at $r<2 \mathrm{kpc}$ only the gas that rotates in the plane of the galaxy is observed. Thus outer HII regions are located inside a broad and thin gaseous ring whose tilt with respect to the main disk increases with radius and reaches $50^{\circ}$ at $r=50^{\prime \prime}(5.8 \mathrm{kpc})$, which corresponds to the $R_{25}$ optical radius. This fact confirms the hypothesis of Whitmore et al. (1990) that Arp 212 is an object "related to polar-ring galaxies". Arp 212 can be safely put into this class of galaxies given the fact that rings in PRG are not always strictly polar- 
warped tilted rings are also commonly found. There is a well-known example - the NGC 660 galaxy, where the outer ring is tilted with respect to the main disk by about $60^{\circ}$ (Arnaboldi \& Galletta, 1993). However, unlike classical PRG, the polar ring in Arp 212 cannot be seen on optical images (except for the HII regions mentioned above) and shows up mostly in neutral hydrogen.

In Fig. 8 we represent the space location of the warped ring with respect to the plane of the galaxy. We constructed the kinematical model solely for ionized gas, although it is evident that we are dealing with a more global gaseous disk interspersed with isolated regions of ongoing star formation. They trace the kinematics of the neutralhydrogen disk with sufficiently high surface density inside the optical radius (Li et al., 1993). Observations at $21 \mathrm{~cm}$ (Li et al.,1993) indicate that we see a single gaseous structure extending from the center to the outermost regions at $r \approx 20-22 \mathrm{kpc}$. Unfortunately, the low angular resolution of HI data prevents a detailed analysis of gas motions outside the optical disk. The only more or less definitive conclusion concerning the kinematics of gas in outer regions based on the data of Li et al. (1993) is that at $r \geq 15 \mathrm{kpc}$ gas rotates about an axis, close to the rotation axis of the galaxy, but the direction of gas rotation is opposite to the rotation of inner regions.

Let us try to extrapolate the behavior of the gaseous disk beyond the optical radius. It is safe to assume that the outer disk (the ring) continues to warp at $r>50^{\prime \prime}$. In this case its plane should become polar to the stellar disk of the galaxy not too far from the center (position (1) in Fig. 8). The continued warp of the gaseous ring at these galactocentric distances is not just an extrapolation of our kinematical model, but is also consistent with the available observational data for PRG with strongly warped disks, e.g., NGC 2655 (Sparke et al., 2008). Such a distortion of the shape of the polar ring is believed to be due to the precession of orbits in a nonspherical gravitational potential. In case of Arp 212, if the warp continues in the same direction, the angle between the orbital plane and the plane of the galaxy should decrease with further increase of $r$, so that the polar ring "tips over" onto the plane of the galaxy (position (2) in Fig. 8). It is also evident from this figure that in case of such a "tipover" the direction of rotation of outer regions should be opposite that of the inner parts of the disk, in accordance with HI observations.

However, there are reasons to doubt the reality of the scenario (2). If the outer gas is located close to the plane of the galaxy then the direction of the orbital momentum should be reversed as a result of precession. It is a very peculiar pattern, because precession is usually a result of withdrawal or redistribution of the angular momentum, whereas in our case we have to invent a mechanism that would transfer substantial angular momentum to gaseous clouds in order to reverse the direction of rotation. It must be a very specific mechanism, because such complex warps of disks (rings) are not commonly observed in PRG. Of course, we can assume that in Arp 212 we observe two independent systems of neutral hydrogen - one of them has produced the polar ring and the other, the counterrotating outer disk. However, we consider it very unlikely to have caught the galaxy during the stage when it interacts simultaneously with two different gaseous subsystems. Moreover, the available HI observations are rather indicative of the existence of a single structure in the distribution of neutral hydrogen.

We therefore consider scenario (1), where outer parts of the HI ring are located in the vicinity of the polar plane, to be more realistic, and, moreover, it is also consistent with the results of observations. Indeed, according to Li et al. (1993), outer HI density contours are appreciably extended along $P A_{2} \approx 25^{\circ}$ and their axial ratio is about $2 / 3$ (see also Fig. 9), which corresponds to $i_{2} \approx 50^{\circ}$ in case of thin-disk projection. We further assume that the Western part of the HI disk is the closest to us and recall that velocities in its Northern half are redshifted, to find from formula (11) that outer parts of the HI ring are located at angle $\delta=82^{\circ}$, and this result agrees well with the polar-disk hypothesis. The weak point of our hypothesis consists in the lack of accurately known kinematical parameters for the gaseous disk at $r>50^{\prime \prime}$. Further highresolution HI data are needed.

Such a behavior of the warped polar ring can be explained by assuming that the distribution of gravitational potential in the galaxy is not spherical. In this case the orbits of the polar component should precess. One of the most famous PRG-NGC 2685, where the HI kinematics can be explained by the deviation of the distribution of potential from spherical symmetry (Józsa et al., 2004)provides a beautiful example of a strong warp of the polar ring. The researchers have repeatedly discussed the stability of gaseous disks in the gravitational potential of threeaxial galaxies. In this case stable are disks that lie in the planes perpendicular to the major or minor axes of the system. The example of the gaseous disk in the elliptical galaxy Cen A analyzed by van Albada et al. (1982) somewhat resembles the warped disk in Arp 212: in the inner region the gaseous disk is orthogonal to the stellar body of the galaxy, but at large radii the gaseous disk in Cen A warps so that its outer parts tend to be located in the rotation plane of the stellar subsystem and rotate in the opposite direction. However, in Arp 212 the situation is quite the opposite - the outer parts of the HI disk are located in the polar plane and with decreasing radius orbits precess and approach the plane of the stellar disk. In any case, the hypothesis that outer parts of the polar ring are located in one of the principal planes of the threeaxial halo (the contribution of the disk to the potential is small at $r=2 R_{25}$ ) appears reasonable, although we bear in mind that the available data are so far insufficient for a definitive conclusion about the three-axial distribution of the potential. Note also that the suggestion for oblate distribution of the potential can be found inside the optical radius. Indeed, it follows from Fig. 4 the velocities of rotation of outer HII regions in the inclined-disk model are somewhat higher than in the inner disk. This is not a 


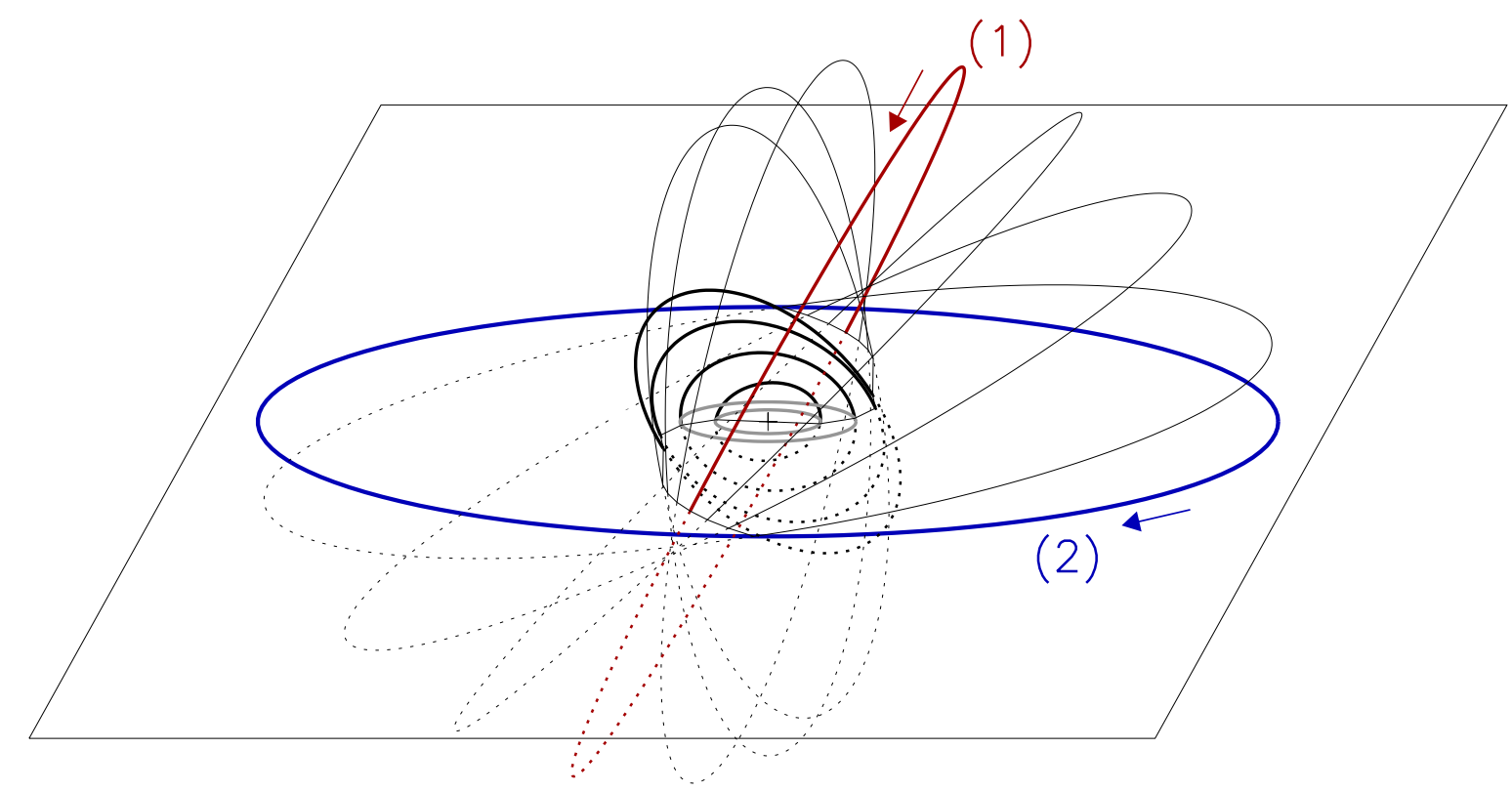

Fig. 8. Orientation of circular orbits in the warped gaseous ring with respect to the main plane of the galaxy. The solid black lines indicate the orbits computed in terms of the two-dimensional model of the velocity field. The thin black lines represent the possible warp of the disk at large galacticentric distances. The gray ellipses are the orbits of the inner disk of ionized gas in the plane of the galaxy. The red and blue ellipses are the two possible orientations of the outer regions of the HI disk ((1) and (2)). The arrows indicate the direction of rotation. The dashed lines correspond to the parts of orbits that lie below the main plane. Inner regions of the main disk rotate in the counterclockwise direction.

surprise, because at these radii an oblate stellar disk must contribute appreciably to the total gravitational potential.

\subsection{The Origin of the Outer Gaseous Subsystem}

According to modern scenarios, the formation of polar rings is due to the interaction between galaxies: their mergers or accretion of the matter of a companion galaxy onto the main galaxy (Bournaud \& Combes, 2003 Combes, 2006). In this case either the companion galaxy or tidal features remaining after its disruption should be found in the neighborhood of the galaxy considered. Above we already pointed out some similarities between Arp 212 and NGC 2655 - another galaxy with a warped polar disk. Deep images of this galaxy, presented by Sparke et al. (2008), show low surface brightness tidal features within two optical diameters. However, our image of Arp 212 lacks tidal features brighter than $25.2^{m} / \square^{\prime \prime}$ (see Section 3).

In Fig. 10 one can see the surface-brightness distribution on the $\mathrm{R}_{c}$ image of the galaxy after the subtraction of the two-dimensional model consisting of a bulge and exponential disk (see Section 3). The distribution of residual surface brightness is mostly due to the star-forming regions and dust lanes in the disk. We failed to find any stellar tidal features associated with a disrupt companion inside the optical radius. The only exception is the "filament" $r=20-40^{\prime \prime}$ North of the nucleus, where one of the outer HII regions projects onto. However, this may be a fragment of the spiral pattern in the stellar disk. The bright dust lane located to the South and Southwest of the nucleus, which coincides with three outer HII regions, is the only structural feature on this image that is undoubtedly associated with the warped polar ring. Here we must be observing dust inside the polar ring. This hypothesis is consistent with our previous conclusion that the Southern half of the ring is the nearest to the observer. This dust lane, like the HII regions, is not indicative of the remnants of a companion, but rather of ongoing star formation inside the polar ring.

The lack of stellar remnants of a dusrupted companion may imply that the polar ring possibly formed as a result of the capture of gas from a companion galaxy. Such a process of the formation of polar rings has been rather thoroughly studied using various methods including numerical simulations (Reshetnikov \& Sotnikova, 1997, Bournaud \& Combes, 2003).

According to Bournaud \& Combes (2003), most of the polar rings form in accordance with the "accretion" scenario. The NED database contains only one galaxyUGC 12549 , inside the region of $1^{\circ}$ radius (414 kpc) centered on Arp 212 within $\pm 1000 \mathrm{~km} / \mathrm{s}$ interval of the lineof-sight velocity of Arp 212. It is an Im-type galaxy, which has almost exactly the same systemic velocity as Arp 212 - $1634 \mathrm{~km} / \mathrm{s}$, but is $3^{m}$ fainter in terms of integrated magnitude. Its distance from Arp 212 (projected onto the sky-plane) is $23.6(163 \mathrm{kpc})$, or $15 D_{25}$. This galaxy is very rich in gas: the total 21 -cm flux of UGC 12549 is 


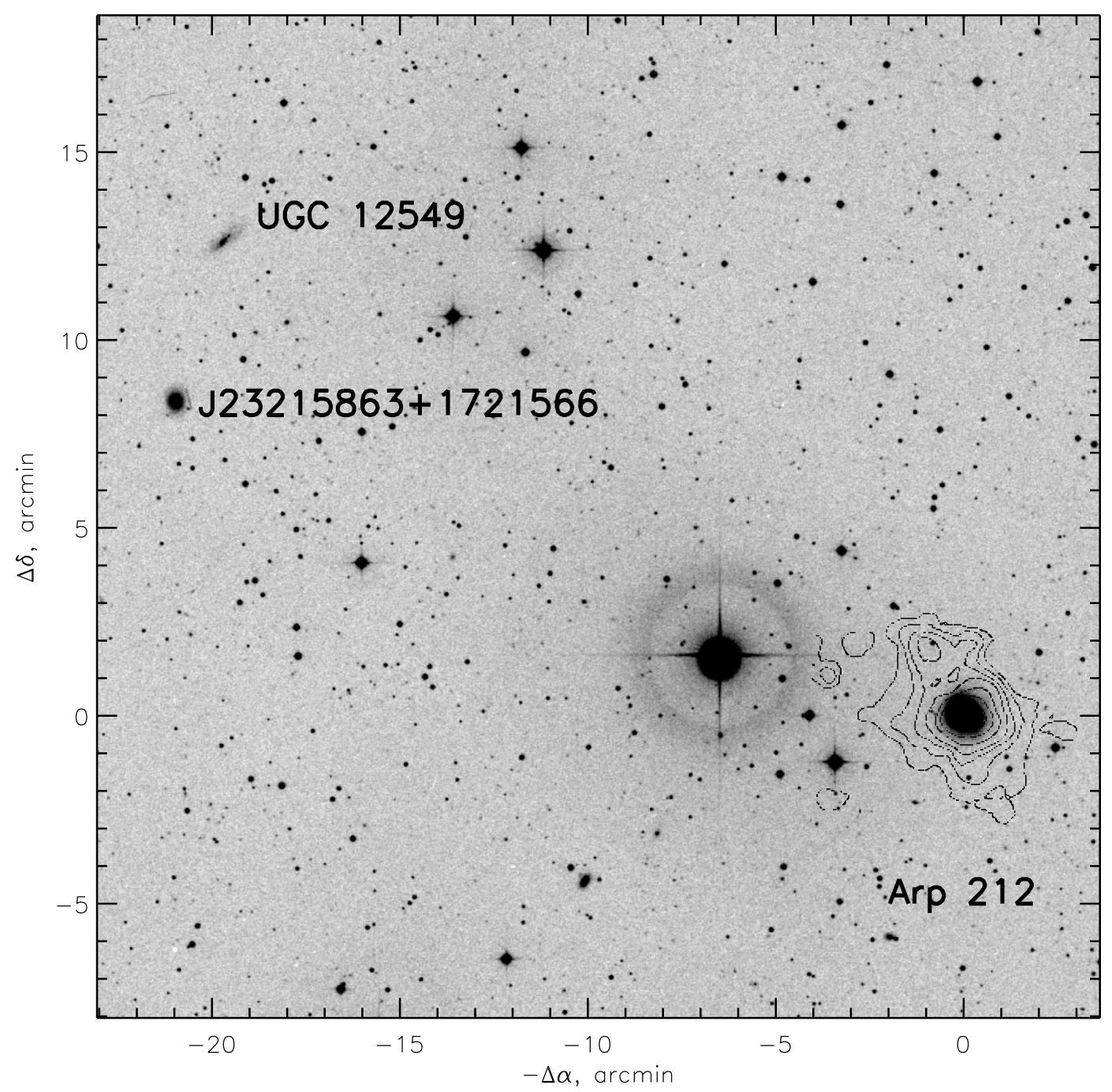

Fig. 9. POSS2 field in the vicinity of Arp 212 with HI density contours (Li et al., 1993) superimposed. Two possible companions are indicated.

only twice lower than that of Arp 212 (Springob et al., 2005). The total HI mass in UGC 12549 then must be $10^{9} M_{\odot}$, and the HI-to-the-total-mass ratio in this galaxy must be several times higher than in Arp 212, where it is about $10 \%$. In Fig. 9 we show both galaxies according to the Palomar DSS2. There is a brighter galaxy, 2MASX J23215863+1721566, with unknown redshift 4.5 $(31 \mathrm{kpc})$ South of UGC 12549. These two galaxies may form a pair. It is evident from Fig. 9 that HI density contours (i.e., the projection of outer regions of the polar ring) in Arp 212 are extended in the direction of this pair of galaxies. There may be a faint tidal tail between the galaxies. However, so far we have no data about the distribution of neutral hydrogen beyond $20-30 \mathrm{kpc}$ from the center of Arp 212. New observations are required in order to confirm or disprove the hypothesis that UGC 12549 served as a donor for the formation of the polar ring in Arp 212.

In conclusion we address the problem of the burst of star formation in Arp 212. According to photometric data, all star-forming regions in the galaxy are $6.1 \pm 0.6 \mathrm{Myr}$ old (Martínez-Delgado, 2007), i.e., the most recent burst of star formation started almost simultaneously throughout the entire galaxy. The dynamical time scale (orbital period) for outer emission filaments is $0.2-0.3 \mathrm{Gyr}$, and that of the outer regions of the HI disk, $0.5-1$ Gyr. It is safe to assume that the outer gaseous subsystem-after it was captured by the main galaxy - formed the polar disk whose gas began, as a result of orbital precession, to "fall" onto the plane of the stellar disk of the galaxy. After 
sufficient amount of gas was accumulated, a burst of star formation began. The fact that the age of the burst of star formation is smaller that the dynamical age of the gaseous disk may imply that such bursts may have occurred more than once in the galaxy considered.

\section{Conclusion}

We constructed and analyzed the velocity field of ionized gas in the peculiar galaxy Arp 212 and found it to have two kinematically distinct subsystems of rotation gasthe inner disk and outer HII regions. The inner disk is located within $3.5 \mathrm{kpc}$ of the center and ionized gas in this disk rotates in the plane coincident with the plane of the stellar disk. Note that the inner part of the ionized-gas disk is exactly coincident with the earlier known moleculargas disk. The second subsystem of ionized gas is located at a galactocentric distance of $r=2-6 \mathrm{kpc}$ and consists of isolated HII regions whose orbits are tilted by significant angles to the stellar disk. Our own and published data on the kinematics of molecular, ionized, and neutral gas can be explained in terms of the following model. Most of the HI mass in the outer parts of the galaxy concentrates in a broad ring with radius of about $20 \mathrm{kpc}$. Outer ring regions rotate in the plane that is orthogonal to the plane of the stellar disk. With decreasing galactocentric distance the orbits of gaseous clouds precess and approach the plane of the disk. This precession is due to the nonspherical (maybe a triaxial) distribution of the gravitational potential in the galaxy. A burst of star formation takes place in the inner regions of the warped polar ring. The angle between the ring and the plane of the galaxy is equal to $50^{\circ}$ at $r=R_{25}$, it continues to decrease with decreasing radius, and at $r \approx 2-3 \mathrm{kpc}$ the gas from the ring falls onto the plane of the galaxy. The powerful dust ring in the central region of the galaxy is indicative of the fall of gas from the polar ring onto the plane of the stellar disk of Arp 212. The polar ring may have formed as a result of the interaction with the gas-rich dwarf galaxy UGC 12549, however, new observations of the distribution and kinematics of neutral hydrogen are required to either confirm or disprove this scenario.

Acknowledgements. This work is based on observations collected with the 6-m telescope of the Special Astrophysical Observatory (SAO) of the Russian Academy of Sciences (RAS), operated under the financial support of the Science Department of Russia (registration number 01-43). This research has made use of the NASA/IPAC Extragalactic Database (NED) which is operated by the Jet Propulsion Laboratory, California Institute of Technology, under contract with the National Aeronautics and Space Administration. I am grateful to Vladimir Resehtnikov for a number of valuable comments that he made after reading the draft of the paper. This work was supported by the Russian Foundation for Basic Research (project no. 06-02-16825) and grant no. MK1310.2007.2 of the President of the Russian Federation.

\section{References}

Afanasiev V.L., Moiseev A.V., 2005, Astronomy Letters, 31, 193 ; astro-ph/0502095

Arnaboldi M., \& and Galletta G., 1993, A\&A, 268, 411

Arp H., 1966, ApJS, 14, 1

Begeman K.G., 1989, A\&A, 223, 47

Bournaud F., \& Combes F., 2003, A\&A, 401, 817

Brosch N., \& Loinger F., 1991, A\&A, 249, 327

Cairós L.M., Vílchez J.M., Gónzalez-Pérez J.N., et al., 2001a, ApJS, 133, 321

Cairós L.M., Caon B., Vílchez J.M., et al., 2001b, ApJS, 133, 321

Coccato L., Corsini E.M., Pizzella A., \& Bertola F., 2007, A\&A, 465, 777

Courteau S., 1997, AJ, 114, 2402 (

Combes F., 2006, in Mass Profiles and Shapes of Cosmological Structures, Eds.: Mamon G.A., Combes F., Deffayet C., Fort B., (EAS Publications Series, 20, p. 97; astro-ph/0508434

Demoulin M.-H., 1969, ApJ, 157, 69

García-Lorenzo B., Cairós L.M., Caon N. et al., 2008, ApJ, 677, 201

Józsa G., Oosterloo T., \& Klein U., 2004, in Proceedings of "Baryons in Dark Matter Halos", Novigrad, Croatia, 2004, Eds.: Dettmar R., Klein U., Salucci P., (Published by SISSA, Proceedings of Science), p. 108

Li J.G., Seaquist E.R., Wrobel J.M. et al., 1993, ApJ, 413, 150

Martínez-Delgado I., Tenorio-Tagle G., Muñoz-Tuñón C., et al., 2007, AJ, 133, 2892

Moiseev A.V., 2002, Bull. SAO 54, 74; astro-ph/0211104

Moiseev A.V., \& Egorov O., Astrophysical Bulletin, 2008, 63, 193; arXiv:0805.2367] [astro-ph]

Moiseev A.V., \& Mustsevoi V.V., 2000, Astronomy Letters, 26, 565; astro-ph/0011225

Moiseev A.V., Valdés J.R. \& Chavushyan V.H., 2004, A\&A, 421, 433

Reshetnikov V.P., Hagen-Thorn V.A. \& Yakovleva V.A., 1993, A\&A, 278, 351

Reshetnikov V., \& Sotnikova N., 1997, A\&A, 325, 933

Shalyapina L.V., Merkulova O.A., Yakovleva V.A., \& Volkov E.V., 2007, Astronomy Letters, 33, 520, 33, 585

Sil'chenko O.K., \& Afanasiev V.L., 2004, AJ, 127, 2641

Sparke L.S., van Moorsel G., Erwin P., \& Wehner E.M.H., 2008, AJ, 135, 99

Springob C.M., Haynes M.P., Giovanelli R., \& Kent B.R., 2005, ApJS, 169, 149

Thuan T.X., \& Martin G.E., 1981, ApJ, 247, 823

van Albada T.S., Kotanyi C.G., \& Schwarzschild M., 1982, MNRAS, 198, 303

Whitmore B.C., Lucas R.A., McElroyet D.B. et al., 1990, AJ, 100, 1489

Yasuda N., Fujisawa K., Sofue Y. et al., 1992, PASJ, 44, 1 


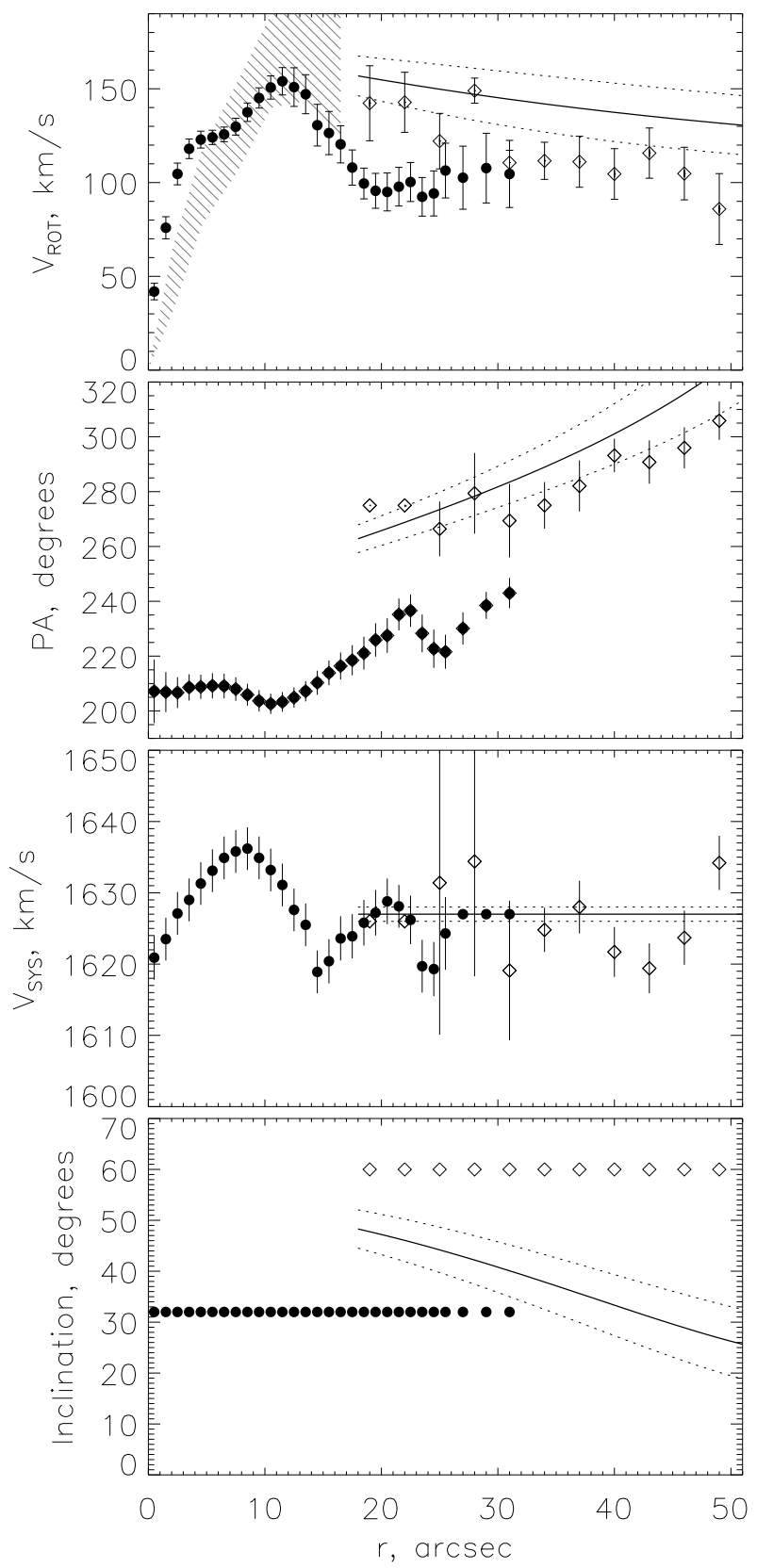

Fig. 4. Radial variations of kinematic parameters characterizing the velocity field of ionized gas (from top to bottom): circular-rotation velocity; position angle, and inclination. The filled circles and open diamond signs correspond to the inner disk and outer emission regions, respectively. The hatched domain on the upper plot reflects the rotation velocity of molecular gas (Li et al., 1993) with the measurement errors taken into account. The solid lines correspond to the kinematical parameters computed in terms of a two-dimensional warped-disk model (see Section 5). The dashed lines indicates the $3 \sigma$ interval computed from errors of model parameters from Table 2 ,

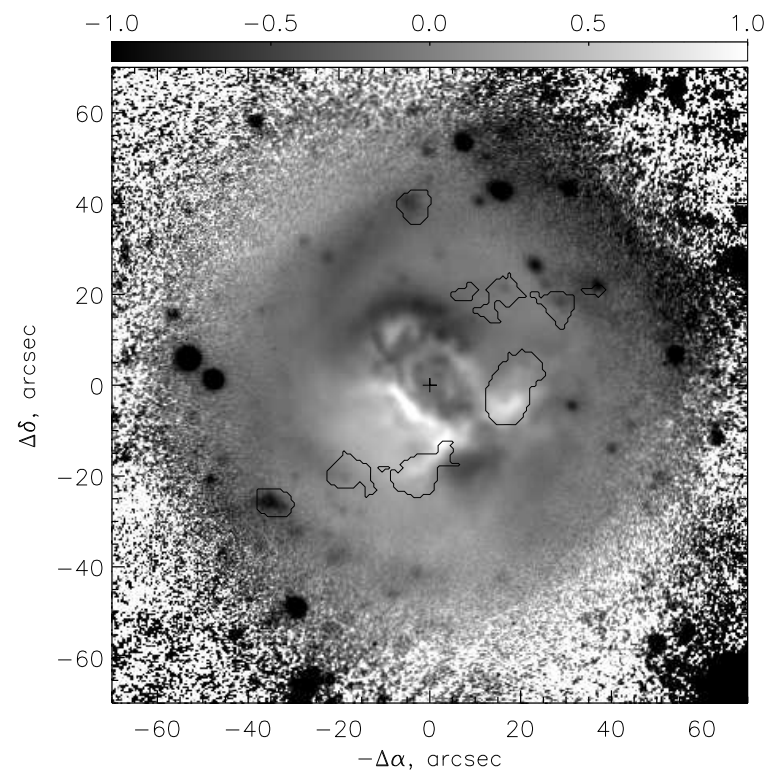

Fig. 10. Residual brightness in the magnitude scale (observations minus the bulge and disk model). The contours indicate the locations of the outer HII regions that belong to the polar ring. 